\title{
Zebrafish: An Emerging Model System in Toxicological Studies
}

\author{
Kamal Kishor* , K. Shrman, R. P. Singh, N. S. Meena, \\ Neelam Dinodia and Rituraj Kewat
}

\section{Department of Veterinary Pharmacology and Toxicology, College of Veterinary Science and A.H. (NDVSU) Jabalpur, Madhya Pradesh (India)}

*Corresponding author

\section{A B S T R A C T}

\section{Keywords}

Zebrafish, Rapid embryonic development, Transparency, Efficient animal model, Pathophysiology similar to humans

Article Info

Accepted:

07 January 2021 Available Online: 10 February 2021
Zebrafish is the suitable model organism for identification of diseases which have same patho-physiology as in humans. A Zebrafish larva absorbs small drug molecules diluted in the surrounding water through their skin and gills hence drug administration is simple as compare to other experimental animals. Yolk sac, sinus venosus or circulation are also act as route of drug administration for the highly hydrophobic compounds, large molecules and proteins. Zebrafish can be used for each assay and small amounts of drug are required as compared to testing in other animal models. Due to transparency of zebrafish for several days' post-fertilization enables in-vivo observation of live or whole mount fixed specimens including the visualization of vital dyes fluorescent tracers, antibodies and riboprobes. Organization of Economic Co-operation and Development (OECD) recommend zebrafish for aquatic toxicity testing. For the assessment of environmental toxicity agents fish have been used for years. Zebrafish have become a smart vertebrate model for toxicological testing. This is much faster, cheaper and more efficient animal model. Its biology has been shown to be highly similar to that of mammals.

\section{Introduction}

The zebrafish over the last decades has developed into an important model organism for toxicological research. The main focus of zebrafish research has been traditionally on developmental biology, keeping and observing zebrafish in the lab led to the identification of diseases similar to humans such as cancer which subsequently became a subject for study. The zebrafish model, with its specific experimental advantages and characteristics has created its own niche in cancer research complementing existing models (Feitsma et al., 2008). The zebrafish, because of its small size, low cost, diverse adaptability, short breeding cycle, high fecundity, ex-utero development of the embryo and transparent embryos has been widely used as a prominent model organism in different fields. It shares a high degree of homology with the human genome. Thus, the zebrafish is becoming a powerful model organism for studying genetics (Kawahara et 
al., 2009), development, toxicology, pharmacology, DNA damage repair, cancer and other diseases (Dai et al., 2014). Zebrafish can be used for eco-environmental monitoring and multitudinous pollutant evaluations studies such as toxic heavy metals, organic pollutants and endocrine disruptors. As zebrafish is sensitive to different contaminants, it is considered an ideal model organism for environmental monitoring. Goolish et al., (2000) studied the characteristic changes in morphology, gene expression, behavior and physiology as biological indicators. The International Organization for Standardization (ISO) first published the zebrafish toxicity test in 1984 . The toxicity models used in these studies ranged from biomolecules such as peptides and proteins, membranes, organelles and cells to multi-cellular organisms such as daphnia and sea urchin and animal models such as mice and rodents. They have a short generation time of 3 months and give prodigiously hundreds of offspring per female per week. Embryos develop externally can be easily genetically manipulated and are transparent. Many factors suggest that the zebrafish is a powerful tool to study human diseases. Useful model systems have many common properties. Zebrafish fill a niche that provides a standardized model species filling the gap between invertebrate and microbial models and the more complex and expensive mammalian model species (Lin et al., 2013). Zebrafish offer many technical advantages that allow rapid experimental progress. One of the many important aspects of zebrafish development is that it can develop relatively normally without any functional blood circulation for up to a week. This allows the researcher to continue experiments well after the compound being examined interferes with or completely blocks heart function. Experiments that could not be accomplished with animals that require cardiac function for continued survival and development can be conducted in zebrafish (Heideman et al., 2005).

Zebrafish begin to swallow at 72 hours postfertilization, so drugs can be delivered orally and absorbs small molecules diluted in the surrounding water through their skin and gills. Highly hydrophobic compounds, large molecules and proteins can be injected into the yolk sac, the sinus venosus or the circulation. In addition to the transparency of zebrafish for several days of post-fertilization enables in-vivo observation of live or whole mount fixed specimens including the visualization of vital dyes fluorescent tracers, antibodies and riboprobes. After 120 hours post-fertilization (hpf), zebrafish develop discrete organs and tissues. These organs and tissues have been found to be similar to their mammalian counterparts at the anatomical, physiological and molecular levels. Drugscreening assays using zebrafish are becoming increasingly popular (Haldi et al., 2006). This convenient predictive animal model can serve as an intermediate step between cell-based evaluation and conventional animal testing (Mcgrath et al., 2008).

\section{Advantages of zebrafish as an animal model for toxicity testing}

Zebrafish are easy to maintain and have large number of offspring (approx. 200 eggs per female)

Zebrafish have rapid embryogenesis (6 days) and generation cycle (2-3 months)

Have well characterized developmental stages, transparent embryo and internal organs can be visualized during embryogenesis

Embryos are permeable to small molecules and large or lipophilic molecules can be microinjected as well as embryos can be directly used to monitor water, soil and waste water samples 
Zebrafish and mammals exhibit a high degree of similarity as it relates to molecular mechanisms of development and cellular physiology

Due to fertilization and development occurs externally, permitting direct observation and manipulation of embryos in a wide variety of laboratory conditions

Easy access for drug administration and vital dye staining and low space requirement on per animal basis (Lele and Krone, 1996).

\section{Procurement of zebrafish}

Adults of zebrafish were purchased from different "research center on zebrafish" in all over the India for the experimental studies. Than according to Bilotta et al, 1999 go for the establishing and maintaining a low cost zebrafish breeding system. Provide the entire necessary requirement and better environment for reproduction of zebrafish due to rapid embryogenesis and generation cycle we got large numbers of zebrafish after some months.

\section{Applications of zebrafish model}

Zebrafish have been used to test the toxicity of synthetic chemicals and natural products since 1950. The toxic effects of organic solvents (like phenol, aniline and cyclohexane) and some metals (like zinc, selenium, mercury and copper) assessed in zebrafish (Vitozzi and De Angelis, 1991). In experiments performed using zebrafish, lethality and malformation were used as general parameters for assessing toxicity.

Due to convenience and low cost of maintaining and handling zebrafish subsequent studies in all areas of toxicology were performed in order to understand the adverse effects of chemicals and to predict results in humans. Studies demonstrated in zebrafish are amenable to chemical screens and exhibit good dose responsiveness to toxicity. In addition, the zebrafish embryo may have a predictive value similar to mammalian teratogenicity and toxicity assays (Ensenbach and Nagel, 1995) (Table 1 and 2).

\section{Eco-toxicity}

Eco-toxicological studies are performed to detect, manage and monitor the presence of environmental pollutants in water, soil and waste. Studies using zebrafish have been developed to directly monitor water, soil and waste-water quality. Zebrafish have also been used to assess risks, predict hazards associated with petroleum products, byproducts and evaluate working conditions in the petrochemical and mining industries. In addition, pesticides, polychlorinated biphenyls, dioxin and some of their derivatives have been studied in zebrafish embryos (Andreasen et al., 2002). Due to rapid development many toxicological endpoints can be evaluated few days after fertilization.

According to Schulte and Nagel (1994) toxicological endpoints at $72 \mathrm{hpf}$ fall into two major groups. Type-I endpoints are used for general morphological evaluation of development and it include egg coagulation, embryo gastrulation, somite formation, tail extension, heart function, spontaneous movement and hatching frequency. Type-II endpoints are more detailed and it include quantification of number of somites, bloodcirculation, eye development, spontaneous movement at $24 \mathrm{hpf}$, cardiac function measured by heart rate, otolith development, melanocyte development, skeletal malformation and delayed hatching (Zhu and Shi, 2002).

\section{Carcinogenicity}

A lots of zebrafish tumor models have been described and new models are being 
generated in a large number of laboratories. However, investigators have exploited the capable of being easily led of zebrafish to explore a host of molecular and cellular pathways implicated in drug-induced carcinogenesis. The zebrafish may also provide a useful model for the discovery of carcinogenicity through the development of chromosomal injury reporters in different sensitized strains or through global assays of chromosomal disruption based on nextgeneration sequencing technologies (Taylor et al., 2009). Carcinogenesis induced by fluorescent genetically labeled human breast cancer cells. Culture MDA-MB-231human breast cancer cells at $37{ }^{\circ} \mathrm{C}$ in DMEM-high glucose medium containing L-glutamine, 10 per cent fetal bovine serum and 1:100 penicillin-streptomycin. Point the needle tip at the injection site and gently insert the needle tip into the perivitelline space between the yolk sac and the periderm of the zebrafish embryo. Inject approximately 400 labeled tumor cells. Make sure that the yolk sac is not ruptured to avoid implantation into the yolk sac. Screen each fish under a fluorescence stereomicroscope at $2 \mathrm{~h}$ post-injection (hpi) for the perivitelline space injection. Remove the embryos with injection errors, such as ruptures or injections of the yolk sac and pick out embryos with injected cells below or above threshold. Keep only the embryos with approximately 400 cells in culture. After crossing parental zebrafish overnight, zebrafish embryos were collected the following morning and were maintained at 28 degree Celsius. The embryos were dechorionated with fine tweezers under stereomicroscope $48 \mathrm{hpf}$. The labeled breast cancer cells were collected and re-suspended in a small amount of PBS. After wellpreparation, suspended cells were loaded into one needle. Approximately 400 cells were injected into the duct of Cuvier (Doc) of the perivitelline space under a stereomicroscope. During the interval, the embryos were subjected to the designed treatment. Cancer cell dissemination by perivitelline space injection or invasion by Doc injection was detected, counted and imaged by confocal microscopy 3 or 6 days post injection (dpi) (Ren et al., 2017).

\section{Hepatotoxicity}

The developing zebrafish can be utilized for hepatotoxicity screening which is amenable to hepatotoxicity applications because of their transparency, speed of development, large sample size and low cost. Hepatotoxicity studies in the zebrafish can therefore be conducted earlier in drug development which may aid the decision-making process during research and development. There are a number of early indicators of liver toxicity in zebrafish. Similar to mammals, zebrafish respond to xenobiotics and oxidative stress with induction of phase-I and phase-II drug metabolism genes. Furthermore, there is a high degree of similarity between zebrafish and human cytochrome $\mathrm{P}_{450}$ (CYP) gene sequences and protein function and the majority are expressed throughout development which allows developing zebrafish to be used for toxicological and pharmacological studies (Peterson et al., 2012).

\section{Cardio-toxicity}

The zebrafish has emerged as an excellent model for studying cardiovascular disease and drug-induced cardiac irregularities such as QT prolongation. Lots of the pharmaceutical industry and regulatory bodies have placed significant emphasis on identifying and eliminating drugs associated with QT prolongation (Hanson et al., 2006). A zebrafish $\mathrm{KCNH}_{2}$ homolog is expressed in both chambers of the embryonic heart and shows high similarity to human $\mathrm{KCNH}_{2}$ particularly in the pore and QT drug-binding 
region. Inhibition of $\mathrm{KCNH}_{2}$ in zebrafish embryos using morpholino antisense oligonucleotides induces bradycardia and a 2:1 atrioventricular block (Arnaout et al., 2007) have identified and characterized two mutant alleles of $\mathrm{KCNH}_{2}$ in zebrafish. ECG recordings in adult zebrafish have demonstrated that QT prolonging drugs cause an increase in corrected QT interval although control drugs do not cause a significant change (Milan et al., 2006).

\section{Ototoxicity}

Zebrafish lateral line hair cells and hair cells of the mammalian inner ear are sensitive to ototoxic drugs such as cisplatin and neomycin. Zebrafish hair cells are easily accessible and can be visualized using fluorescent in-vivo dyes facilitating the development of high-throughput screening assays (Chiu et al., 2008) tested a library of 1040 compounds for ototoxicity in a zebrafish assay. The behaviors including feeding, seizures, involuntary movement, flight responses, sleeping and addiction can be directly observed and quantified in the zebrafish. Similarly, genetic and chemical effects on each of these behaviors in humans can be recapitulated in the zebrafish (Eimon et al., 2009).

\section{Nano-toxicity}

The zebrafish larval bioassay has been used to rapidly evaluate how physical properties such as size, shape, charge and surface chemistry affect toxicity. The nano-material-biological interactions knowledge base is a repository of annotated and integrated data of nanomaterial characterization for which zebrafish have made substantial contributions (Kim et al., 2013).

\section{Visual system of toxicity}

The visual system of toxicity in zebrafish is considered to be an especially relevant model for comparisons to humans because of the conserved structure of the retina and the ability of zebrafish to see colors as like mice and rats. Some standardized assays have been developed to measure zebrafish larval eye functions including optomotor response (OMR) and optokinetic response (OKR). The OMR assay measures movement of the large zebrafish eyes and the OKR assay measures locomotion in response to visual cues (Richards et al., 2008).

\section{Reproductive toxicity}

In-vitro fertilization and embryogenesis make the zebrafish a simpler and more attractive animal model for investigating reproductive toxicity. Most structural classes of toxicants have been evaluated for reproductive toxicity in zebrafish, including metals, organochlorines, pesticides, halogenated aromatic hydrocarbons, synthetic and natural estrogens and other industrial chemicals. The index suggested by OECD 229 for assessing reproductive toxicity using zebrafish is focused on the gonad growth index, reproduction ability, sperm quality, vitellogenin (Vtg) expression assay (Wang et al., 2011).

\section{Developmental toxicity}

The zebrafish has included a large number of non-regulatory studies in which mortality and common morphological defects of developmental toxicity and teratogenicity in zebrafish are assessed through bright-field microscopy. These defects include morphological abnormalities, altered hatching, altered body size, eye size, head size, formation of body curvature, tail formation, pigmentation, swim-bladder inflation, edema and malformation in the pericardial and yolk sac (He et al., 2014). Many compounds that affect hatching either delay or shorten zebrafish hatching. For 
example, shortened hatching time has been reported for the insecticide methoxychlor, acetone and 1per cent dimethylsulfoxide (DMSO), the latter being one of the most common solvents for chemicals when testing in zebrafish. Two common endpoints for developmental toxicity testing are body size and curvature. Changes in body curvature include axial curvature, dorsal curvature, altered tail formation and lordosis (Ducharme et al., 2013).

Table.1 Toxicity of various compounds in zebrafish

\begin{tabular}{|c|c|c|}
\hline Compound & Activity & Observed toxicity \\
\hline Amifostine & Radio protector & Swim bladder \\
\hline Cyclosporin-A & Immunosuppressive drug & $\begin{array}{l}\text { Teratogen, liver, } \\
\text { gastrointestinal }\end{array}$ \\
\hline Doxorubicin & Anticancer drug & $\begin{array}{l}\text { Teratogen, } \\
\text { cardiovascular }\end{array}$ liver, kidney, \\
\hline Dexamethasone & Corticosteroid & Gastrointestinal, liver, kidney \\
\hline Fluorouracil & Anticancer drug & Liver, kidney \\
\hline Methotrexate & Anticancer drug & $\begin{array}{l}\text { Teratogen, liver, kidney } \\
\text { gastrointestinal }\end{array}$ \\
\hline
\end{tabular}

(Kari et al., 2007)

Table.2 The analysis of metal, metal oxide and carbon-based nanoparticles

\begin{tabular}{|l|l|l|l|ll|}
\hline \multicolumn{1}{|c|}{ Nano-particles } & \multicolumn{1}{|c|}{ Stage } & \multicolumn{1}{|c|}{ LC $_{\mathbf{5 0}} \mathbf{( m g / L )}$} & \multicolumn{1}{c|}{$\begin{array}{c}\text { Time } \\
(\mathbf{h r s} .)\end{array}$} & \multicolumn{1}{|c|}{ Effects } \\
\hline Silver & Eggs & 2.7 & 48 & Malformations \\
\hline Gold & Eggs & Less than 200 & 48 & No effect \\
\hline Cadmium & Eggs & $186(\mathrm{nM})$ & 120 & $\begin{array}{l}\text { Delayed hatching and } \\
\text { Malformations }\end{array}$ \\
\hline Copper & $\begin{array}{l}\text { Eggs } \\
\text { Adults }\end{array}$ & $\begin{array}{l}24 \\
4.2\end{array}$ & 48 & $\begin{array}{l}\text { Delayed hatching and } \\
\text { Malformations } \\
\text { No effect }\end{array}$ \\
\hline Titanium dioxide & $\begin{array}{l}\text { Eggs } \\
\text { Adults }\end{array}$ & $\begin{array}{l}\text { Less than } 1600 \\
\text { Less than } 1600\end{array}$ & 48 & $\begin{array}{l}\text { Premature hatching } \\
\text { No effect }\end{array}$ \\
\hline Zinc oxide & Eggs & 1.8 & 96 & Delayed hatching \\
\hline Magnesium oxide & $\begin{array}{l}\text { Eggs } \\
\text { Adults }\end{array}$ & 428 & 96 & Delayed hatching \\
\hline Fullerene & Eggs & 1.5 & 96 & Reduce hatching \\
\hline
\end{tabular}

(Haque and Ward, 2018)

\section{Endocrine disruption}

The chemicals which may mimic or interfere with the endocrine processes in humans and animals such substances have been termed endocrine disruptors (EDCs). Estrogens and their analogs are the most potent endocrine disruptors identified. OECD recommends the use of several fish species for monitoring estrogens and estrogen analogs. Endocrine disruption testing and assessment (EDTA) studies may produce more accurate and predictive results through zebrafish model than other species (Sumpter and Jobling, 1995). An adult zebrafish model shows the transfer kinetics and trans-generational effects 
in offspring due to chronic exposure of bisphenol-A (BPA). Thyroid endocrine disruption and developmental neurotoxicity were observed in larval offspring by parental exposure to BPA alone or in combination with titanium dioxide nanoparticles (n-TiO2). Parental exposure of bisphenol-A alone or combined with $\mathrm{n}-\mathrm{TiO} 2$ results in developmental toxicity in their offspring, significant delay hatching rates, increase in malformations was observed at $5 \mathrm{dpf}$. Survival rate are also significantly decreased. In females, the $\mathrm{T} 4$ concentration was significantly lower in the $20 \mu \mathrm{g} / \mathrm{L} \mathrm{BPA}$ treatment group than the control group. Decreased plasma T4 or T3 levels were observed in adult zebrafish after exposure to $20 \mu \mathrm{g} / \mathrm{L}$ BPA with or without $100 \mu \mathrm{g} / \mathrm{L} \mathrm{n}$ $\mathrm{TiO} 2$, indicating the thyroid endocrine disrupting effects of BPA in fish. Both the reduced thyroid hormone levels along-with the transfer of BPA and n-TiO2 to eggs and larvae may impair the development of the central nervous system (Guo et al., 2019).

\section{Disposal of experimental zebrafish}

All the animals (zebrafish) used in experiment were collected and sacrificed, because live animal may cause cross contamination in their surroundings. These animals were sacrificed with minimum pain and suffering, after euthanizing them with tricaine methane sulfonate according to method described by Matthews and Varga, 2012. Sacrificed animals were disposed off as bio hazardous waste.

\section{Future applications}

Zebrafish serves as a useful platform for researchers interested in processes such as different types of toxicity study and drug development. For cancer biologists it opens a window onto longitudinal analysis of tumor growth, invasion, metastasis and angiogenesis at an anatomic resolution not readily achievable in murine or other systems. The zebrafish will allow for detailed in-vivo study of stem and cancer biology in a vertebrate organism with great relevance to human biology (White et al., 2008). Now a days various molecular biology techniques and zebrafish model transgenic lines have been developed for this purpose. Various zebrafish microarrays and huge genomic resources are now a days available for nano-toxicity evaluation. All these advance resources makes zebrafish an extremely multipurpose system for toxico-genomic studies of nanomaterial in the very near future (Chakraborty et al., 2016).

In conclusion the fish have been used for years to assess the toxicity of environmental agents. Zebrafish have become a smart vertebrate model for toxicological testing. This is much faster, cheaper and more efficient animal model. Its biology has been shown to be highly similar to that of mammals. It is an excellent animal model to understand the various steps involved in animal model development and validating with known compounds in order to gain confidence and experience in animal model establishment.

Zebrafish provides the way for the minimization of the usage of mammals in teaching and toxicological research. The zebrafish is uniquely suited to contribute novel insights in cancer biology and provide a 'whole-organism test tube' for the rapid identification of cancer markers, the assessment of their functions, the study of host responses and the development of anticancer drugs. Zebrafish has been shown to be a predictive animal model for assessing drug toxicity and safety. Assessment of a large numbers of compounds from diverse drug classes is necessary to further validate the zebrafish model. 


\section{Acknowledgements}

The author is extremely thankful to Dr. Y.P. Sahni, Director Research Services, Nanaji Deshmukh Veterinary Science University, Jabalpur, Madhya Pradesh for going through the manuscript. The authors are also gratefully acknowledged Dr. Neha Shukla for very valuable guidance and kind support.

\section{References}

Andreasen, E.A., Spitsbergen, J.M., Tanguay, R.L., Stegeman, J.J., Heideman, W. and Peterson, R.E. Tissue-specific expression of AHR2, ARNT2 and CYP1A in zebrafish embryos and larvae: effect of developmental stage and 2,3,7,8-tetrachlorodibenzo-p-dioxin exposure. Toxicological Science. 2002; 68: 403-419.

Arnaout, R., Ferrer, T. and Huisken, J. Zebrafish model for human QT syndrome. Procedure of National Academy Science USA. 2007; 104(27): 1316-1321.

Bilotta, J., Saszik, S., Amy, S., Delorenzo and Hardesty H.R. Establishing and maintaining a low-cost zebrafish breeding and behavioural research facility. Behavior Research Methods, Instruments and Computers. 1999; 31(I): 178-184.

Chakraborty, C., Sharma, A.R., Sharma, G. and Lee, S.S. Zebrafish: a complete animal model to enumerate the nanoparticle toxicity. Journal of Nanobiotechnology. 2016; 14(65): 1-13.

Chiu, L.L., Cunningham, L.L. and Raible, D.W. Using the zebrafish lateral line to screen for ototoxicity. Journal of Association Research on Otolaryngology. 2008; 9(2): 178-190.

Dai, Y.J., Jia, Y.F., Chen, N., Bain, W.P., Li, Q.K., Ma, Y.B., Chen, Y.L. and Pei, D.S. Zebrafish as a model system to study toxicology. Environmental Toxicology and Chemistry. 2014; 1(33): 11-17.

Ducharme, N.A., Peterson, L.E., Benfenati, E., Reif, D., McCollum, C.W. and Gustafsson, J.A. Meta-anlysis of toxicity and teratogenicity of 133 chemicals from zebrafish developmental toxicity studies. Reproductive Toxicology. 2013; 41: 98108.

Eimon, P.M. and Rubinstein, A.L. The use of in-vivo zebrafish assays in drug toxicity screening. Expert Opinion Drug Metabolism and Toxicology. 2009; 5(4): 393-401.

Ensenbach, U. and Nagel, R. Toxicity of complex chemical mixture, acute and long- term effects on different life stage of zebrafish. Ecotoxicological Environmental Safety. 1995; 30: 151157.

Feitsma, H. and Cuppen, E. Zebrafish as cancer model. Molecular Cancer Research. 2008; 6(5): 685-694.

Goolish, E.M., Okutake, K. and Johnson, P. The behavioural response of zebrafish to hypergravity conditions. Journal of Gravitational Physiology. 2000; 7: 99100.

Guo, Y., Chen, L., Wu, J., Hua, J., Yang, H., Yang, L., Wang, Q., Zhang, W., Lee, J. and Zhon, B. Parental co-exposure of bisphenol-A and nono-TiO2 causes thyroid endocrine disruption and developmental neurotoxicity in zebrafish offspring. Science of the Total Environmental. 2019; 650: 557-565.

Haldi, M. Human melanoma cells transplanted in to zebrafish proliferate, migrate, produce melanin, form masses and stimulate angiogenesis in zebrafish. Angiogenesis. 2006; 9: 139-151.

Hanson, L.A., Bass, A.S. and Gintant, G. ILSI-HESI cardiovascular safety subcommittee initiative: evaluation of 
the three non-clinical models of QT prolongation. Journal of Pharmacological and Toxicological Methods. 2006; 54(2): 116-129.

Haque, E. and Ward, A.C. Zebrafish as a model to evaluate nanoparticle toxicity. Molecular Diversity Preservation International. 2018; 561(8): 1-18.

He, J.H., Gao, J.M., Huang, C.J. and Li, C.Q. Zebrafish model for assessing developmental and reproductive toxicity. Neurotoxicology and Teratology. 2014; 42: 35-42.

Heideman, W., Antikiewich, D.S., Carney, S.A. and Peterson, E. Zebrafish and cardiac toxicology. Cardiovascular Toxicology. 2005; 2(5): 203-214.

Kari, G., Rodeck, U. and Dicker, A.P. Zebrafish: an emerging model system for human disease and drug discovery. Nature. 2007; 1(82): 70-80.

Kawahara, A., Nishi, T., Hisano, Y., Fukui, H., Yamaguchi, A. and Mochizuki, N. The sphingolipid transporter spns2 functions in migration of zebrafish myocardial precursors. Science. 2009; 323: 524-527.

Kim, K.T., Zaikova, T., Hutchison, J.E. and Tanguay, R.L. Gold nanoparticles disrupt zebrafish eye development and pigmentation. Toxicological Science. 2013; 133(2): 275-288.

Lele, Z. and Krone, P.H. The zebrafish as a model system in developmental, toxicological and transgenic research. Biotechnology Advances. 1996; 1(14): 57-72.

Lin, S., Zhao, Y., Nel, A.E. and Lin, S. Zebrafish: an in-vivo model for nano EHS studies. Small. 2013; 10(9): 16081618.

Matthews and Varga. Anesthesia and Euthanasia in Zebrafish. ILAR Journal. 2012; 53(2): 192-204.

McGrath, P. and Li, C.Q. Zebrafish: a predictive model for assessing drug- induced toxicity. Drug Discovery Today. 2008; 10(13): 394-401.

Milan, D.J., Jones, I.L., Ellinor, P.T. and Macrae, C.A. In-vivo recording of adult zebrafish electrocardiogram and assessment of drug induced QT prolongation. American Journal of Physiology Heart Circulatory Physiology. 2006; 291(1): 269-273.

Peterson, R.T. and Macrae, C.A. Systematic approaches to toxicology in the zebrafish. The Annual Review of Pharmacology and Toxicology. 2012; 52: 433-453.

Ren, J., Lui, S., Cui, C. and Dijke, P. Invasive behaviour of human breast cancer cells in embryonic zebrafish. Journal of Visualised Experiments. 2017; 122: 1-9.

Richards, F.M., Alderton, W.K. and Kimber, G.M. Validation of the use of zebrafish larvae in visual safety assessment. Journal of Pharmacological and Toxicological Methods. 2008; 58(1): 50-58.

Schulte, C. and Nagel, R. Test acute toxicity in the embryo of zebrafish, Brachydanio rerio, as an alternative to the acute fish test: preliminary results. Alternative of Lab Animals. 1994; 22: 12-19.

Sumpter, J.P. and Jobling, S. Vitellogenesis as a biomarker for estrogenic contamination of the aquatic environment. Environment Health Prospect. 1995; 103: 173-178.

Taylor, A.M. and Zon, L. Zebrafish tumor assays: the state of transplantation. Zebrafish. 2009; 6: 339-346.

Vitozzi, L. and De, A. A critical review of comparative acute toxicity data on fresh water fish. Aquatic Toxicology. 1991; 19: 167-204.

Wang, R.L., Bencic, D., Lazorchak, J., Villeneuve, D. and Ankley, G.T. Transcriptional regulatory dynamics of the hypothalamic- pituitary-gonadal axis and its peripheral pathway as 
impacted by the Ecotoxicological and Environmental Safety 3-beta HSD inhibitor trilostane in zebrafish. 2011; 74(6): 1461-1470.

White, R.M., Sessa, A., Burke, C., Bowman, T., Leblance, J., Ceol, C., Bourque, C.,
Dovey, M., Goessling, W., Burns, C.E. and Zon, L. Transparent adult zebrafish as a tool for in-vivo transplantation analysis. Cell Stem Cell Resource. 2008; 2: 183-189.

\section{How to cite this article:}

Kamal Kishor, K. Shrman, R. P. Singh, N. S. Meena, Neelam Dinodia and Rituraj Kewat. 2021. Zebrafish: An Emerging Model System in Toxicological Studies. Int.J.Curr.Microbiol.App.Sci. 10(02): 826-835. doi: https://doi.org/10.20546/ijcmas.2021.1002.098 